\title{
Lingue: beni collettivi immateriali, che spesso, e per fortuna, si materializzano
}

Claudio Marazzini

PUBBLICATO: 31 MARZO 2020

Il giorno 5 di ottobre del 2019 si sono celebrati a Udine i roo anni della Società Filologica Friulana. Per l'occasione, il presidente dell'Accademia della Crusca, Claudio Marazzini, è stato invitato a tenere una relazione, nel contesto di una serie di interventi rivolti al pubblico che gremiva il bellissimo Salone del Parlamento della Patria del Friuli. Gli atti della giornata saranno pubblicati nella gloriosa rivista "Ce fastu?". Intanto, però, il prof. Federico Vicario, presidente della Società Filologica Friulana, su richiesta del prof. Marazzini, ha permesso che questo intervento fosse anticipato in una pubblicazione dell'Accademia della Crusca. In questo modo si ribadisce il legame stretto tra le due Accademie, con l'auspicio di una collaborazione fattiva e fruttuosa.

I titolo del mio intervento si collega alla definizione, ormai abbastanza comune, delle lingue intese come "beni collettivi immateriali", adoperata sempre più frequente negli ultimi anni non soltanto nelle celebrazioni ufficiali, sui giornali o nella divulgazione più o meno raffinata, ma anche presente nei testi specialistici dei giuristi e nelle comunicazioni burocratiche e ministeriali. Vorrei riflettere su questo tema: se la collocazione della lingua, anzi delle lingue in genere, di qualunque lingua, allinterno di questa categoria classificatoria sia pienamente soddisfacente, e se sia davvero vantaggiosa per le lingue stesse.

Vedremo innanzitutto di ricapitolare le circostanze che hanno reso cosi popolare e diffusa la categoria dei "beni culturali immateriali". Se non l'origine, almeno la formalizzazione concettuale deriva dalla scelta di un organismo internazionale qual è l'UNESCO. Il patrimonio culturale immateriale dell'Unesco possiede oggi addirittura un logo, che qui riproduco:

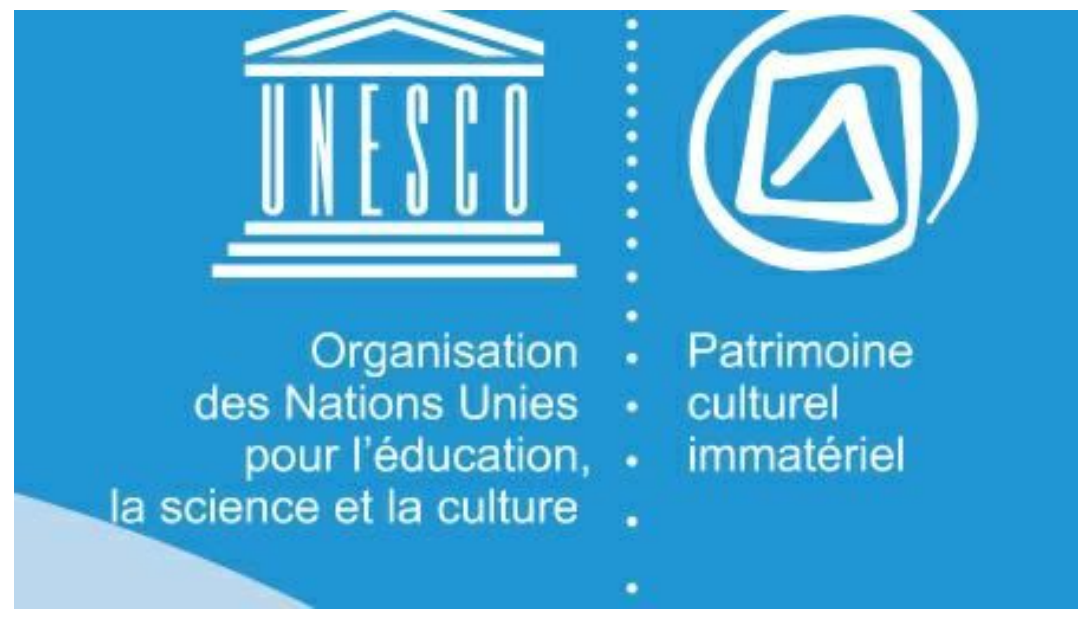

Abbiamo anche una precisa data di riferimento, il 2003; è l'anno della Convenzione per la salvaguardia del patrimonio culturale immateriale. Il testo originale, in data 17 ottobre 2003 , è in francese, non in inglese, perché la convenzione è stata elaborata a Parigi, in un contesto in cui la Francia è riuscita ancora da dare spazio alla propria lingua. Naturalmente il testo è stato poi tradotto in inglese, e qui si può immediatamente cogliere una differenza terminologica, perché al patrimoine culturel inmatériel del testo originale corrisponde in inglese intangible cultural heritage, che può essere abbreviato nella sigla ufficiale ICH. Nella trasposizione inglese compare il termine heritage, che si differenzia non poco dal concetto dipatrimoine del testo francese. La terminologia italiana è meno univoca, perché fa 
riferimento ai beni culturali immateriali, ma anche al patrimonio culturale immateriale, calco sul francese, con sigla PCI. A volte si usa anche ereditá culturale immateriale, calco del cultural heritage inglese.

Tendenzialmente, il concetto di bene culturale immateriale sembra avere guadagnato terreno sulle forme concorrenti, tanto è vero che nel Supplemento dell'Enciclopedia italiana del 2015 un'esperta del settore, Roberta Tucci, ha compilato, sotto la voce Beni culturali, la sottovoce Beni culturali immateriali, dalla quale si possono ricavare alcune informazioni interessanti, a cominciare dalla cronologia. La studiosa afferma infatti che l'espressione beni culturali immateriali (per i linguisti potrebbe essere definita una combinazione lessicale o un'espressione polirematica che dir si voglia) è entrata in uso nella seconda metà degli anni 'go come evoluzione del concetto di "beni culturali", che ovviamente esisteva già prima; inoltre la studiosa aggiunge che in italiano esisteva il concetto di beni culturali volatili, circoscritto a un ambito disciplinare piu ristretto (la formula, di Alberto M. Cirese, risale al I988), mentre il concetto di beni materiali ha segnato una nuova fase, nella quale l'uso di un linguaggio standardizzato ha consentito la condivisione ad ampio raggio internazionale, in diretta connessione con la terminologia dell'Unesco. Infatti l'aggettivo immateriale, come dicevamo, traduce l'inglese intangible di intangible heritage, anche se sarei portato ad affermare che non è assente un riflesso del francese originale immatériel. La cronologia indicata dalla studiosa può essere confermata dalla proiezione di questo grafico ricavato da NGram Viewer:

\section{Google Books Ngram Viewer}

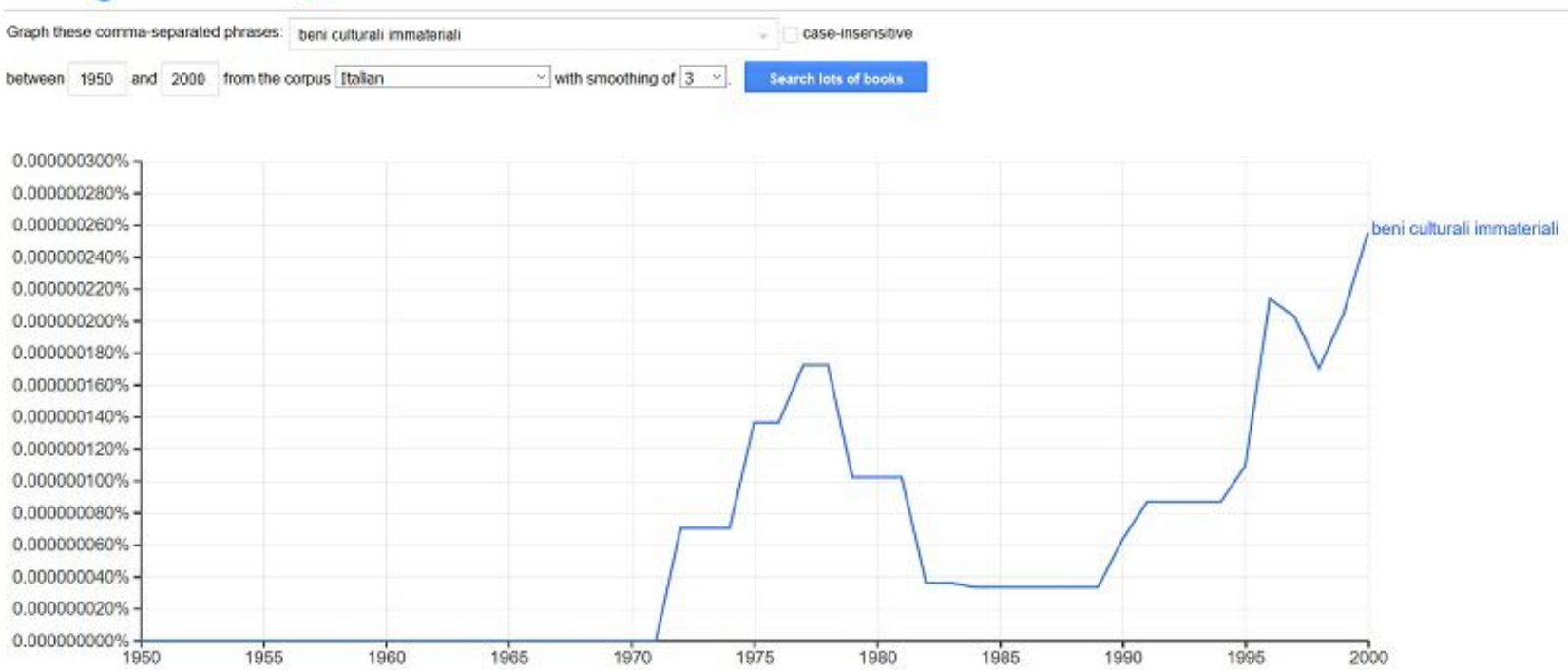

Come molti sanno, NGram Viewer (uno degli strumenti di Google) permette di costruire grafici su base statistica, ricavando i dati dal grande patrimonio di Google Books. Il limite di questa ricerca sta nel fatto che essa si arresta all'anno 2000 e non va oltre; ma la tesi della professoressa Tucci, secondo la quale la forte circolazione della combinazione lessicale beni culturali immateriali è collocabile nell'ultimo decennio del secolo scorso, sembra in ogni modo confermata dal grafico, cosi come quest'altro grafico, sempre tratto da NGram Viewer, mostra la lenta ma costante crescita nell'uso italiano della polirematica beni culturali, iperonimo statisticamente ricorrente, come ovvio, in maniera molto più intensa rispetto ai beni culturali materiali: 


\section{Google Books Ngram Viewer}

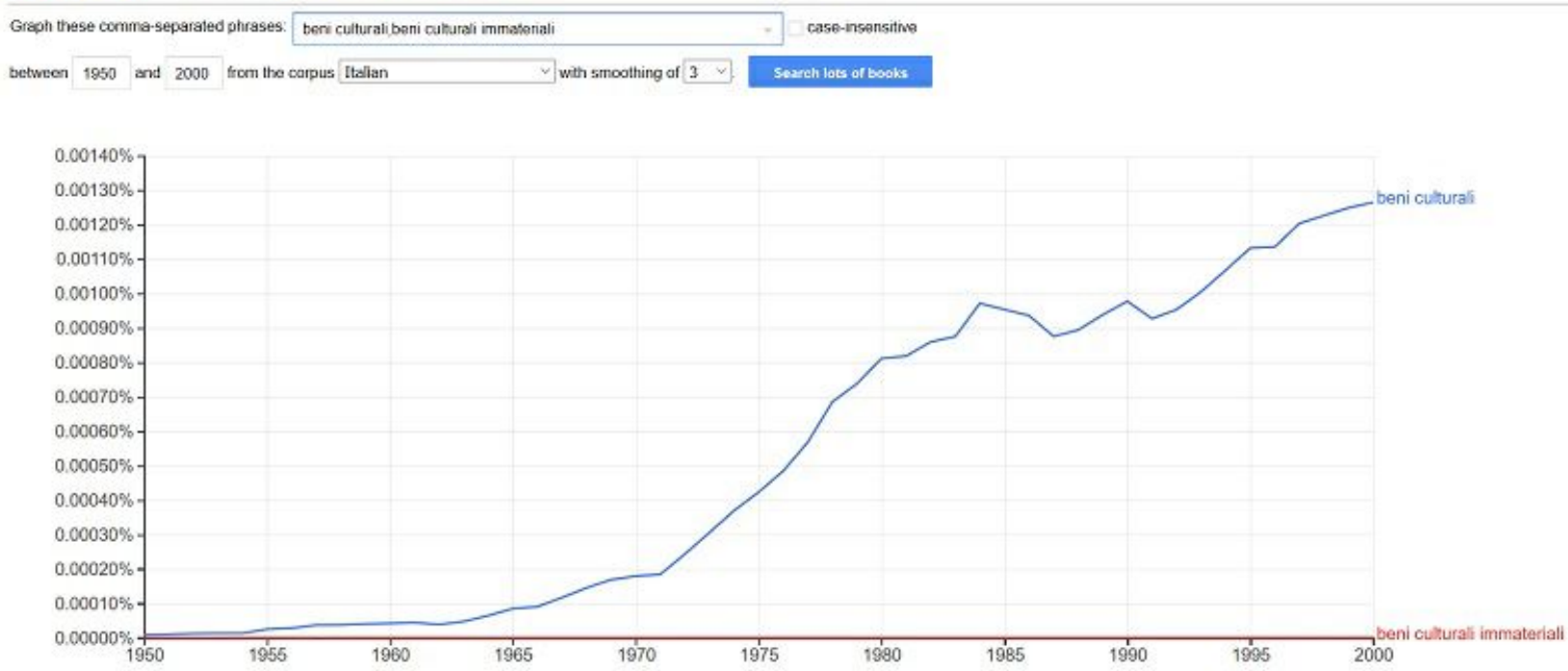

Dagli anni '6o si assiste alla fortuna della polirematica beni culturali, in riferimento, naturalmente, ai beni culturali tradizionali, artistici archeologici e archivistici.

Nonostante il sintagma beni culturali immateriali assuma il valore di effettiva espressione polirematica, in quanto combinazione lessicale stabile, essa stenta a essere riconosciuta dalla lessicografia. Si veda ad esempio il vocabolario Treccani del 1987 (uso l'edizione elettronica disponibile on line nel sito http://www.treccani.it/vocabolario/vocabolario/) :

\section{immateriale}

\begin{tabular}{ll}
\hline Vocabolario on line & \\
\hline Crea un ebook con questa voce / Scaricalo ora $(0)$ & Condividi $y$ G* in
\end{tabular}

immateriale agg. [dal lat. tardo immaterialis, comp. di in ${ }^{2}$ e materialis «materiale»]. - Che non è materiale, che non è formato di materia: forma, sostanza $i$.; capitale $i$., nel linguaggio econ., l'ingegno, la capacità professionale o l'abilità tecnica di una persona in quanto possa essere fonte di ricchezza; in diritto, cose o beni i., quelli che non hanno un'entità materiale o sensibile, pur avendo un contenuto patrimoniale (per es., i prodotti dell'ingegno umano nelle svariate forme della produzione scientifica, artistica o letteraria). Con sign. estens., che oltrepassa i limiti della materia, che appare quasi inconsistente o spiritualizzato: delicatezza, bellezza immateriale. Avv. immaterialménte, non materialmente.

Questa voce lessicografica è stata scritta alle soglie degli anni '9o, dunque proprio all'inizio del processo di crescita, e qui si vede che il concetto di immateriale, predicato per i beni culturali immateriali, non si riferisce ancora a quell'àmbito demo-etno-antropologico, che invece rappresenta il grande ampliamento concettuale determinato della scelta dell'UNESCO. Il riferimento, invece, va al linguaggio del diritto, e i beni immateriali sono quelli che non hanno entità materiale o sensibile, pur avendo un contenuto patrimoniale: sono i prodotti dell'ingegno, scientifici, artistici o letterari. La mancanza del significato più recente è perfettamente giustificabile in un dizionario della fine degli anni 
'8o, ma non è tuttavia superata anche dalla lessicografia più moderna. Ecco la voce dello Zingarelli 2020 , in cui si fa riferimento al bene immateriale in quanto bene incorporale, cioè assolutamente slegato dalla materialità:

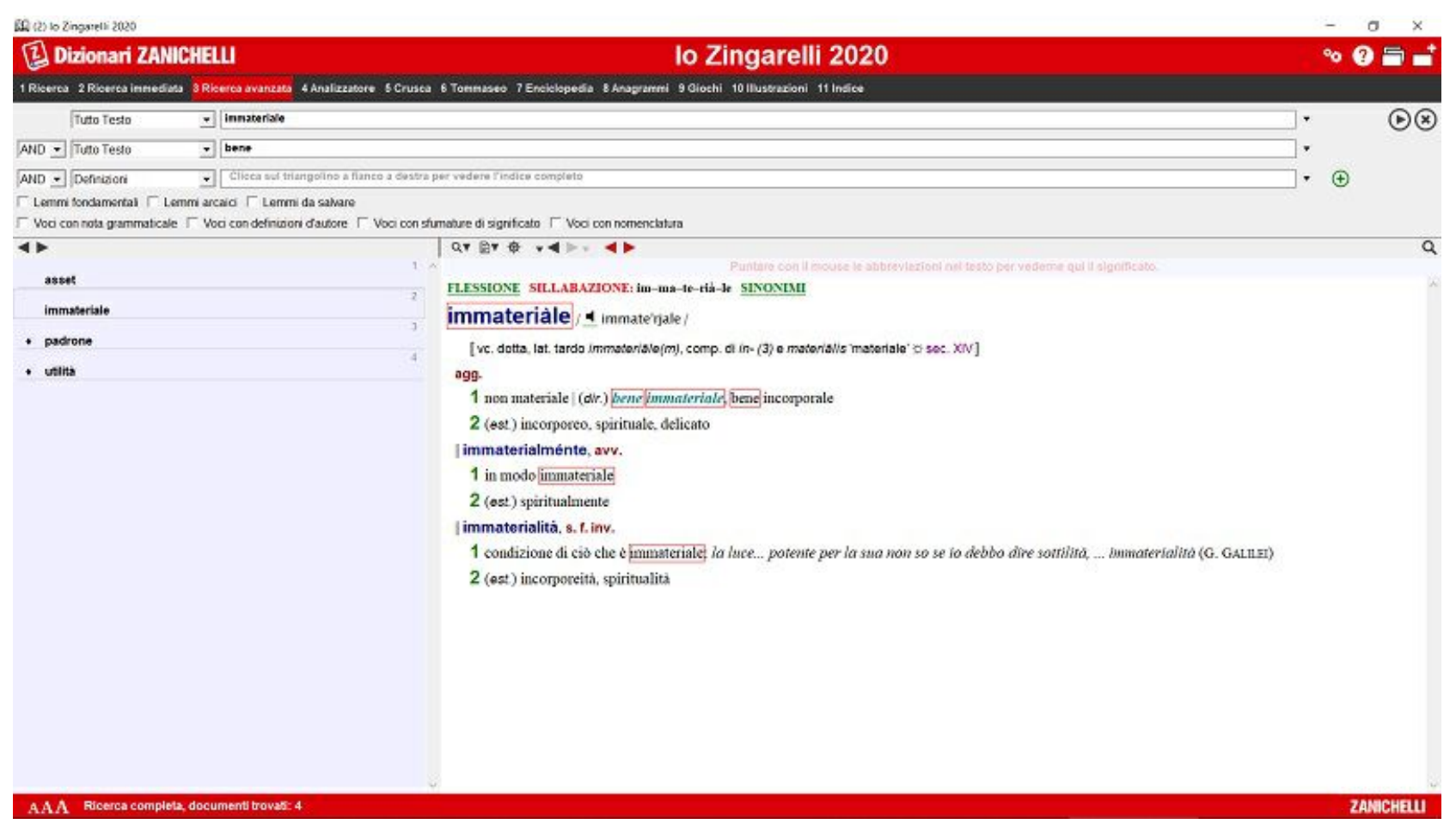

Vedremo tra poco che, nella concettualizzazione dell'UNESCO, la materialità è invece presente in maniera molto forte. Leggiamo infatti l'articolo 2 della Convenzione per la salvaguardia del patrimonio culturale immateriale dell'Unesco:

Article 2 : Définitions

Aux fins de la présente Convention,

I. On entend par "patrimoine culturel immatériel" les pratiques, représentations, expressions, connaissances et savoir-faire - ainsi que les instruments, objets, artefacts et espaces culturels qui leur sont associés - que les communautés, les groupes et, le cas échéant, les individus reconnaissent comme faisant partie de leur patrimoine culturel. Ce patrimoine culturel immatériel, transmis de génération en génération, est recréé en permanence par les communautés et groupes en fonction de leur milieu, de leur interaction avec la nature et de leur histoire, et leur procure un sentiment d'identité et de continuité, contribuant ainsi à promouvoir le respect de la diversité culturelle et la créativité humaine. Aux fins de la présente Convention, seul sera pris en considération le patrimoine culturel immatériel conforme aux instruments internationaux existants relatifs aux droits de l'homme, ainsi qu'à l'exigence du respect mutuel entre communautés, groupes et individus, et d'un développement durable.

2. Le "patrimoine culturel immatériel", tel qu'il est défini au paragraphe I ci-dessus, se manifeste notamment dans les domaines suivants :

(a) les traditions et expressions orales, y compris la langue comme vecteur du patrimoine culturel immatériel ;

(b) les arts du spectacle;

(c) les pratiques sociales, rituels et événements festifs;

(d) les connaissances et pratiques concernant la nature et l'univers;

(e) les savoir-faire liés à l'artisanat traditionnel.

$[\ldots]$ 
L'articolo si compone di due commi. Il primo definisce il concetto di patrimonio culturale immateriale, il secondo elenca in maniera specifica, analiticamente, quanto può essere riconosciuto come parte di tale patrimonio. Il secondo comma ci interessa in modo speciale, perché in esso compare esplicitamente il riferimento alla lingua, intesa però come vettore del patrimonio culturale immateriale, quindi non di per sé stessa, ma come strumento per qualcos'altro. Gli esempi delle scelte compiute nel tempo dall'UNESCO, che vedremo tra poco, potranno chiarire in che senso si intenda l'espressione vettore. Il testo iniziale è francese, ma il testo inglese del medesimo articolo 2 ci presenta la solita equivalenza tra patrimonio culturale immateriale e intangible cultural heritage. Emerge anche il problema della corrispondenza tra langue francese e language inglese:

\section{Article 2 - Definitions}

For the purposes of this Convention,

I. The "intangible cultural heritage" means the practices, representations, expressions, knowledge, skills as well as the instruments, objects, artefacts and cultural spaces associated therewith - that communities, groups and, in some cases, individuals recognize as part of their cultural heritage. This intangible cultural heritage, transmitted from generation to generation, is constantly recreated by communities and groups in response to their environment, their interaction with nature and their history, and provides them with a sense of identity and continuity, thus promoting respect for cultural diversity and human creativity. For the purposes of this Convention, consideration will be given solely to such intangible cultural heritage as is compatible with existing international human rights instruments, as well as with the requirements of mutual respect among communities, groups and individuals, and of sustainable development.

2. The "intangible cultural heritage", as defined in paragraph I above, is manifested inter alia in the following domains:

(a) oral traditions and expressions, including language as a vehicle of the intangible cultural heritage;

(b) performing arts;

(c) social practices, rituals and festive events;

(d) knowledge and practices concerning nature and the universe;

(e) traditional craftsmanship.

[...]

Per chiarire in che modo si traduca in pratica la concettualizzazione dell'Unesco, si possono verificare gli elementi italiani riconosciuti fino al 2018 come meritevoli di entrare nella lista del patrimonio culturale immateriale. Vediamo questi elementi, con a sinistra la data in cui sono entrati nell'elenco (arretrando dal 2018 al 2008). Ne fanno parte l'opera dei pupi siciliani, il canto a tenore sardo, la fabbricazione dei violini di Cremona, la dieta mediterranea (elemento transnazionale, che comprende anche altri paesi, Cipro, Croazia, Grecia, Marocco, Spagna, Portogallo), gli apparati portati a spalla nelle grandi feste, soprattutto religiose (con un elenco di alcune di queste feste in Italia), la coltivazione della vite a Pantelleria, la falconeria (di nuovo un elemento transazionale), l'arte del pizzaiolo napoletano (ne parlarono molto i giornali, interpretando in forma riduttiva e un po' fuorviante, cioè dicendo che la pizza napoletana era diventata patrimonio dell'UNESCO), l'arte dei muretti a secco (di nuovo transnazionale):

2018

Representative List of the Intangible Cultural Heritage of Humanity

Art of dry stone walling, knowledge and techniques Croatia - Cyprus - France - Greece - Italy Slovenia - Spain - Switzerland

2017

Representative List of the Intangible Cultural Heritage of Humanity Art of Neapolitan 'Pizzaiuolo' Italy 
2016

Representative List of the Intangible Cultural Heritage of Humanity

Falconry, a living human heritage Germany - Saudi Arabia - Austria - Belgium - United Arab

Emirates - Spain - France - Hungary - Italy - Kazakhstan - Morocco - Mongolia - Pakistan -

Portugal - Qatar - Syrian Arab Republic - Republic of Korea - Czechia

2014

Representative List of the Intangible Cultural Heritage of Humanity

Traditional agricultural practice of cultivating the 'vite ad alberello' (head-trained bush vines) of the community of Pantelleria Italy

\section{3}

Representative List of the Intangible Cultural Heritage of Humanity

Celebrations of big shoulder-borne processional structures Italy

Mediterranean diet Cyprus - Croatia - Spain - Greece - Italy - Morocco - Portugal

2012

Representative List of the Intangible Cultural Heritage of Humanity

Traditional violin craftsmanship in Cremona Italy

2008

Representative List of the Intangible Cultural Heritage of Humanity

Canto a tenore, Sardinian pastoral songs Italy

Opera dei Pupi, Sicilian puppet theatre Italy

Questi esempi mostrano non soltanto l'evidente prevalenza dell'elemento demo-etno-antropologico nella pratica del patrimonio culturale immateriale, ma mostrano anche la forte presenza di qualche cosa che a stento può essere concepito come immateriale. In molti di questi elementi la materialità è determinante, e l'immaterialità va dunque riferita solo alle abilità di chi costruisce il manufatto materiale sulla base di una tradizione ereditata dal passato, come cultural heritage. Per verificare se le nostre osservazioni sono corrette, esploreremo la lista degli elementi selezionati dall'UNESCO cercando, questa volta in tutti i paesi, nell'arco temporale 2018-2008, non solo in Italia, quali di essi contengano effettivamente elementi di natura prettamente linguistica. La ricerca è facilitata per mezzo di un motore di interrogazione, perché nel sito dell'Unesco esiste un motore di ricerca che permette di esplorare con facilità il patrimonio (https://ich.unesco.org/en/lists). Secondo la mia ricerca, l'elemento prettamente linguistico si riduce a ben poco, una volta che si siano accantonate le varie forme di canto e di spettacolo (spettacolo teatrale parlato o narrazione tradizionale):

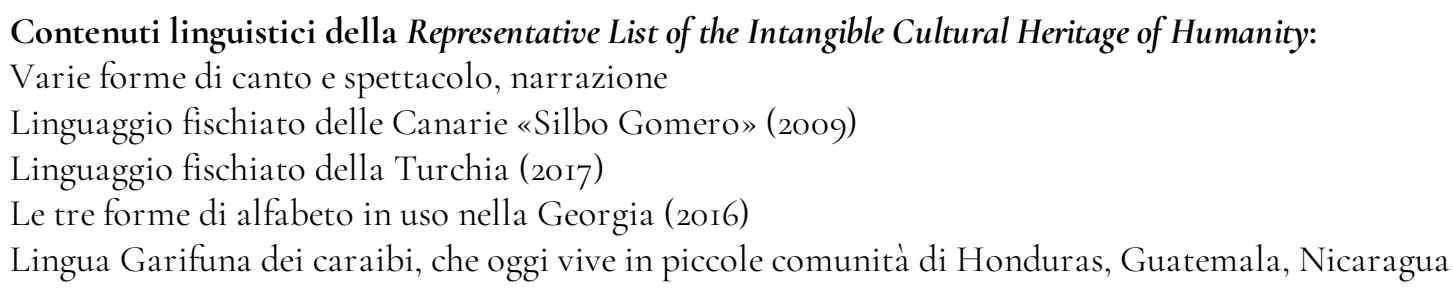

Le forme di spettacolo sono ben rappresentate, ma naturalmente la lingua in questo caso è semplicemente veicolo di altro. Più specifici sono i "linguaggio fischiati" delle Canarie e della Turchia, e interessanti sono le tre forme di alfabeto in uso in Georgia. Infine vi è una lingua vera e propria, seppur estremamente minoritaria: la lingua Garifuna dei Caraibi, che sopravvive in piccole comunità di Honduras, Guatemala e Nicaragua. 
Rispetto alle scelte complessive, queste presenze linguistiche si caratterizzano prima di tutto per il valore demo-etno-antropologico, e poi per la curiosità e rarità. Sono del massimo interesse, certo, ma restano pur sempre molto particolari, estremamente circoscritte, estranee dunque, in sostanza, alla vita delle lingue, ivi comprese soprattutto le lingue minoritarie di maggior peso e respiro. Del resto la difficoltà di introdurre la lingua all'interno delle categorie dell'Unesco si ricava anche da un altro dato, cioè delle risposte al questionario inviato alle Commissioni nazionali dell'Unesco nel 20oo, prima dell'elaborazione del documento finale del 2003:

\section{Definitions for «intangible cultural heritage» - Member States Replies to questionnaires sent to National Commissions in February and August 2000 \\ Questi gli stati che hanno risposto citando in qualche modo la «lingua»: \\ Madagascar, Comunità del Pacifico, Zimbawe, Croazia («language, dialects, tongues, toponymics, and traditional literature»), Lituania, Messico, Venezuela.}

Ogni Stato doveva rispondere indicando il concetto di intangible cultural heritage cosi com'era valutato dalla propria Commissione nazionale. Ebbene, tra tutti i paesi che risposero al questionario, si trova un riferimento specifico alla lingua soltanto nei paesi qui sopra indicati. Come si vede, sono pochissimi, e l'indicazione più precisa e completa viene dalla Croazia, che suggerisce linguaggio, lingua parlata, toponomastica e letteratura tradizionale. Il risultato del questionario giustifica la posizione marginale delle lingue nel documento finale.

Abbiamo parlato a lungo di un rapporto difficile tra il concetto di 'immateriale' e le reali scelte dell'UNESCO, che si traduce nella scelta di testimonianze etno-demo-antropologiche caratterizzate il più delle volte da una forte materialità. Se ne accorsero persino i legislatori italiani, quando, nel 2oo8, il Codice dei beni culturali fu adeguato, inserendo un nuovo articolo, il 7 bis, che faceva riferimento alle indicazioni dell'UNESCO:

Art. 7 -bis. Espressioni di identità culturale collettiva (articolo introdotto dall'art. 1 del d.lgs. $n .62$ del 2008)

Le espressioni di identità culturale collettiva contemplate dalle Convenzioni UNESCO per la salvaguardia del patrimonio culturale immateriale e per la protezione e la promozione delle diversità culturali, adottate a Parigi, rispettivamente, il 3 novembre 2003 ed il 20 ottobre 2005, sono assoggettabili alle disposizioni del presente codice qualora siano rappresentate da testimonianze materiali e sussistano i presupposti e le condizioni per l'applicabilità dell'articolo ro.

Usci fuori una sorta di ossimoro, del resto estremamente significativo: si stabili che le espressioni di identità culturale collettiva contemplate dalle convenzioni UNESCO per la salvaguardia del patrimonio culturale immateriale sarebbero state assoggettabili alla disposizione del Codice soltanto qualora fossero rappresentate da testimonianze materiali. Quindi - questa la conclusione paradossale il patrimonio immateriale ricade sotto la tutela del Ministero dei beni culturali soltanto quando è materiale.

$$
* * *
$$

Alla luce di quanto abbiamo detto, sembra abbastanza difficile riportare in maniera efficace la tutela delle lingue, di qualunque dimensione esse siano, sotto lo scudo costruito dall'UNESCO, che sembra piuttosto estraneo al vero significato di una vera lingua, la quale non può essere considerata soltanto vettore gli altri patrimoni culturali, ma funziona di per se stessa. È vero che le parti della lingua che si traducono in oggetti materiali (prodotti della scrittura, libri, archivi, biblioteche, manoscritti) trovano per fortuna perfetta protezione nelle norme vigenti. La lingua, però, si colloca in un terreno differente, 
perché è altro. Anche l'eventuale differenza tra il cultural heritage e il patrimonio, che appaiono concettualmente non sovrapponibili, spinge a vedere in modo diverso la lingua: essa può essere intesa come eredità, e allora è quella che ci viene trasmessa dai nostri antenati. In tal modo sembra sia prevedibile una protezione in chiave puristica e conservatrice. Invece l'idea di patrimonio può includere anche la capacità di una lingua di adeguarsi ai tempi, di integrare le novità, di accogliere e adattare elementi di origine forestiera, reagendo ad essi. La difesa, in tal caso, potrebbe entrare in gioco nel caso in cui si cerchi volutamente di escluderla o emarginarla, non perché la si voglia modificare. La lingua è al tempo stesso passato, presente e futuro. Ciò rende complesso il rapporto con essa. Nella sua più pura immaterialità, la lingua funziona come sistema grammaticale e sintattico, e come sistema di produzione lessicale, di accettazione o rifiuto di neologismi. Quindi funziona come una macchina: è un meccanismo in perenne attività, e per questo la sua tutela si presenta in modo molto diverso rispetto a tutto il resto. La tradizione consegna agli uomini di oggi elementi, usi e capacità del passato; la lingua viene da lontano, da molto lontano, e porta con sé il proprio passato, ma intanto viaggia verso il futuro.

Tuttavia, stabilite queste differenze, non possiamo negare che il quadro teorico e giuridico del patrimonio immateriale, nato al di fuori della lingua, in sostanza segnato da un passaggio dai pieni poteri dei beni culturali, intesi nel senso artistico tradizionale, verso il nuovo potere dei beni, ora intesi in chiave demo-etno-antropologica, in un quadro comunque largamente insoddisfacente per la lingua, ha dato, nonostante tutto, qualche supporto per aiutare la lingua a resistere di fronte alle aggressioni più gravi. Mi riferisco ovviamente in questo caso alla storia specifica della lingua italiana, e alla sentenza 42/2017 della Corte costituzionale, che ha segnato un passaggio decisivo nella lunga questione giudiziaria provocata dalla scelta del Politecnico di Milano (compiuta nel 20II-20I2) di eliminare la lingua italiana dei corsi avanzati e di dottorato. Non entrerò certo nei dettagli, né voglio ricapitolare qui una vicenda che ha richiesto l'intervento dell'Accademia della Crusca, su cui molti hanno scritto articoli e libri, e che nonostante tutto non si è ancora conclusa ${ }^{\mathrm{I}}$. Allo stato attuale, mi interessa però esaminare alcune delle motivazioni con le quali la suprema Corte ha potuto difendere quella che ha definito la primazia dellitaliano. La suprema Corte non ha potuto fare ricorso a elementi oggettivi esplicitamente presenti nella Costituzione Italiana che evidenziassero un primato della lingua italiana in Italia, anche perché la Costituzione parla di protezione delle lingue solo per quelle di minoranza, e non fa cenno a un'eventuale lingua "ufficiale", esplicitata solo dalla legge 482 del I999, che non è legge costituzionale. Tuttavia, con raffinata mossa giuridica, i giudici hanno ricondotto la primazia dell'italiano all'articolo 9 della Costituzione, proprio l'articolo che parla dei beni culturali; cioè la Corte ha utilizzato indirettamente (senza dichiararlo in forma aperta), e riprendendo in forma piu ampia il concetto di bene culturale immateriale, come era stato elaborato nel corso degli anni, sotto lo stimolo delle indicazioni dell'UNESCO. Un passo di questa lunga e articolata e raffinata sentenza fa riferimento nella prima riga all'articolo 9 , e si chiude con la valorizzazione dell'italiano inteso come bene culturale in sé:

La lingua italiana è dunque, nella sua ufficialità, e quindi primazia, vettore della cultura e della tradizione immanenti nella comunità nazionale, tutelate anche dall'art. 9 Cost. La progressiva integrazione sovranazionale degli ordinamenti e l'erosione dei confini nazionali determinati dalla globalizzazione possono insidiare senz'altro, sotto molteplici profili, tale funzione della lingua italiana: il plurilinguismo della società contemporanea, l'uso d'una specifica lingua in determinati ambiti del sapere umano, la diffusione a livello globale d'una o più lingue sono tutti fenomeni che, ormai penetrati nella vita dell'ordinamento costituzionale, affiancano la lingua nazionale nei più diversi campi. Tali fenomeni, tuttavia, non debbono costringere quest'ultima in una posizione di marginalità: al contrario, e anzi proprio in virtù della loro emersione, il primato della lingua italiana non solo è costituzionalmente indefettibile, bensì - lungi dall'essere una formale difesa di un retaggio del passato, inidonea a cogliere i mutamenti della modernità - diventa ancor più decisivo per la perdurante trasmissione del patrimonio 
storico e dell'identità della Repubblica, oltre che garanzia di salvaguardia e di valorizzazione dell'italiano come bene culturale in sé.

Mi sembra plausibile che i giudici abbiano tratto ispirazione da quanto è stato elaborato dall'UNESCO attorno al tema dei beni culturali immateriali. Anche la parola vettore, che compare nella prima riga di questo estratto della sentenza, richiama un concetto che abbiamo già ritrovato nei documenti dell'UNESCO.

Naturalmente ci si puó chiedere se la via migliore per la tutela delle lingue sia quella seguita dalla Corte costituzionale. Inevitabilmente, ciò è avvenuto in mancanza d'altro, ossia in assenza di una protezione diretta dettata esplicitamente dalla normativa. Altra protezione potrebbe venire da una normativa esplicita: ci sono in Europa varie Costituzioni nazionali che tutelano in maniera più solida la lingua ufficiale delle rispettive nazioni. Il primo posto va alla Francia, ma poi si possono citare le Costituzioni di Spagna e di Portogallo, e la Costituzione della Svizzera ${ }^{2}$. Quest'ultima non colloca la lingua ufficiale e le lingue minoritarie nell'elenco delle lingue da tutelare, non distingue tra questi ruoli, ma si limita a stabilire rapporti tra le lingue che definisce non soltanto ufficiali, ma anche nazionali, termine che rarissimamente ricorre nelle Costituzioni degli Stati. Non voglio concludere, si badi, invocando l'introduzione di una tutela esplicita della lingua italiana all'interno nella Costituzione, cosi come invece la Costituzione già tutela le lingue minoritarie. Non intendo riprendere quella che è stata in passato una proposta venuta dall'Accademia della Crusca attraverso i suoi presidenti. Il mio obiettivo consisteva semplicemente nell'esplorazione del rapporto tra la tutela di una lingua e il concerto moderno di bene culturale immateriale. Abbiamo visto che esso è piuttosto insoddisfacente, anzi evanescente, e si applica con una certa difficoltà alle lingue, specialmente qaruelle di maggior dimensione; tuttavia, in circostanze di emergenza e di conflitto, ha fornito ai giuristi alcuni appigli importanti per resistere alla più grave aggressione condotta negli ultimi anni contro la lingua italiana, e per questo siamo invogliati a esprimere comunque un giudizio positivo, si soddisfazione, seppure solo parziale e condizionata.

Note:

I. Cfr. M.A. Cabiddu (a cura di), L'italiano alla prova dellinternazionalizzazione, Milano, Guerini e associati, 20I7; N. Maraschio, D. De Martino (a cura di), Fuori l'italiano dall'universitá? Bari, Laterza, 20ı2; C. Marazzini, L'italiano è meraviglioso. Come e perché dobbiamo salvare la nostra lingua, Milano, Rizzoli, 20I8, pp. 56-69.

2. Cfr. C. Marazzini, La lingua, il giudice, la costituzione. Una vertenza tutta italiana, e un confronto internazionale, Pisa, Pacini, 2018.

\section{Cita come:}

Claudio Marazzini, Lingue: beni collettivi immateriali, che spesso, e per fortuna, si materializzano, "Italiano digitale", 2020, XII, 2020/1 (gennaio-marzo)

DOI: $10.35948 / 2532-9006 / 2020.3300$

Copyright 2020 Accademia della Crusca

Pubblicato con licenza creative commons CC BY-NC-ND 